In order to obtain more information concerning this question, analogous measurements have been carried out. During three balloon flights (March 26, 1936 and June 18, 1936, results still not published; May 14, 1938) the ionization was explored ${ }^{3}$ between $6 \mathrm{~km}$. and $10 \mathrm{~km}$., within which range Suckstorff has detected the greatest oscillations.

Within the limits of observational errors (generally a few per cent) quite smooth curves have been obtained, displaying no irregularities. The most convincing results were obtained in the last flight, when two ionization pressure chambers were used, filled with krypton and nitrogen, respectively, the specific ionization in nitrogen and krypton being quite different for cosmic and radioactive gamma rays ${ }^{4}$. The velocity of the ascent of the balloons was of the same order of magnitude as in Suckstorff's flights (in the first flight it was even less). The apparatus used were at least twenty times more sensitive than that used by Suckstorff. We are compelled to assume that the oscillations observed by Suckstorff were due to some instrumental faults.

As regards the anomalies observed by J. Juilfs ${ }^{5}$ in his measurements carried out in the lower troposphere (up to the height of $5.7 \mathrm{~km}$.), we agree that radioactive substances of terrestrial origin are not unlikely to cause deviations in the ionization curve from its normal shape. It would be highly desirable to have these interesting observations repeated with modern sensitive instruments.

Physical Laboratory,

S. ZIEMECKI.

Main School of Agriculture,

Warsaw.

April 25.

${ }^{1}$ Kolhörster, W., Naturwis8., 27, 243 (1939).

2 Suckstorff, G. A., Phys. Z., 35, 368 (1934).

${ }^{3}$ Ziemecki, S., and Narkiewicz-Jodko, K., NATURE, 13\%, 944 (1936). Szczeniowski, S.. Ziemecki, S., and Narkiewicz-Jodko, K., NATURE, 142, 208 (1936). For more details, see Bull. Acad. Polon. $A, 318$ (1936), and 273 (1938).

- Juilfs, J., NATURE, 140, 767 (1937). Szczeniowski, S., Ziemecki, S. and Narkiewicz-Jodko, K., Bull. Acad. Polon., A, 273 (1938).

${ }^{5}$ Juilfs, J., Naturwis8., 26, 789 (1938).

Anomaly of the Specific Heat of Potassium Dihydrogen Phosphate at the Upper Curie Point

The dielectric properties of Rochelle salt, potassium dihydrogen phosphate and potassium dihydrogen arsenate, depend on the electric field in the same way that the properties of ferromagnetic substances depend on the magnetic field. The spontaneous polarization is large between the two Curie points and depends on temperature. Approaching the higher (normal) Curie point from lower temperatures, the spontaneous polarization vanishes. As a consequence the specific heat shows an anomaly, corresponding to the magneto-caloric effect in ferromagnetics.

This anomaly was found by Kobeko and Nelidow ${ }^{1}$ and Rusterholz ${ }^{2}$ in Rochelle salt. Wilson ${ }^{3}$ and Hicks and Hooley ${ }^{4}$, however, could not detect it. The variation of the spontaneous polarization as a function of temperature is so slow, that the anomaly of specific heat is spread over a wide temperature rango and therefore the deviations are small. Although the maximum of specific heat measured by Rusterholz was considerably higher than was expected from the theory, the total anomalous heat capacity $\int \Delta C d T$ agrees well with the values calculated from recent results of the spontaneous polarization (Hablützel ${ }^{5}$ ).

As the change of spontaneous polarization of potassium dihydrogen phosphate is larger and more

rapid with temperature, it is probable that the electrocaloric effect will be more pronounced. The specific heat of this salt was therefore measured in a vacuum calorimeter. The salt was heated by a constant flow of radiation and the increase of temperature was measured as a function of time.

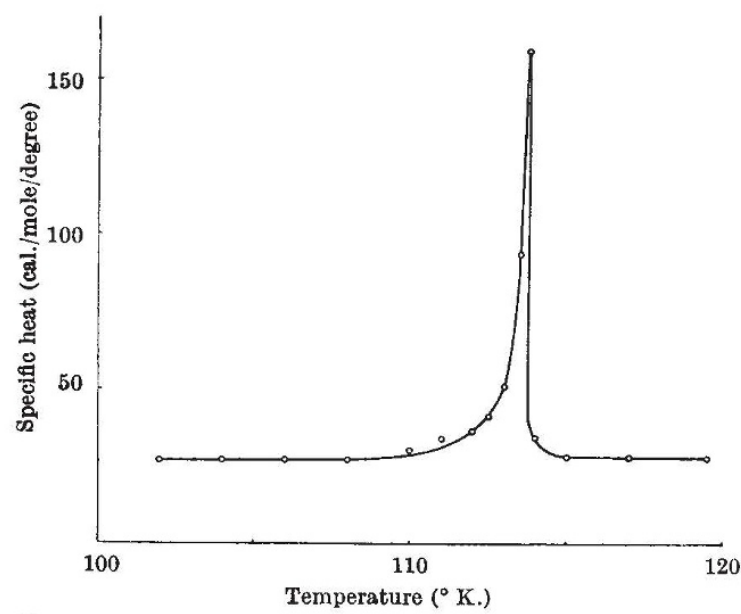

SPECIFIC HEAT OF POTASSIUM DIHYDROGEN PHOSPHATH

The results plotted in the accompanying graph show a strongly marked anomaly of specific heat of about $130 \mathrm{cal} . / \mathrm{mole} /$ degree. The integral $\int \Delta C d T$ representing the energy necessary to destroy the internal field, was found by this method to be 60 cal./mole. With this value and a maximum polarization $P_{\max .}=4.3 \times 10^{-6}$ coulombs $/ \mathrm{cm}^{2}$ measured by Busch $^{6}$, the Lorentz value $f$ is found to be $0 \cdot 5$, applying the Langevin-Weiss formula $\int \triangle C d T=$ $\frac{1}{2} f P^{2}$ max. This is in fair agreement with the value $f=0.4$ calculated by Busch from dielectric investigations.

The variation of the specific heat as a function of temperature can be calculated from the differential Langevin-Weiss formula. Owing to the rapid increase of $P_{\text {spont. as a function of temperature, it is therefore }}$ not possible to give an exact maximum value of $\triangle C$, but the agreement between observed and calculated values is fairly satisfactory. It is intended to investigate potassium dihydrogen arsenate and also the effects at the lower Curie point.

$$
\begin{gathered}
\text { Department of Physics, } \\
\text { Federal Institute } \\
\text { of Technology, } \\
\text { Zurich. } \\
\text { May 4. }
\end{gathered}
$$

W. Bantle.

P. SCHERRER.

Kobeko and Nelidow, Phys. Z . d. Sowjetunion, 1, 382 (1932).

'Rusterholz, Helv. phys. Acta, 8, 39 (1934).

${ }^{3}$ Wilson, Phys. Rev., 54, 1103 (1938).

- Hicks and Hooley, J. Amer. Chem. Soc., 60, 2994 (1938).

${ }^{5}$ Hablützel, Helv. phys. Acta, in the Press.

${ }^{6}$ Busch, Helv. phys. Acta, 11, 269 (1938).

\section{Crystal Structure of Diphenyldiacetylene}

Diphenyldiacetylene $\left(\mathrm{C}_{6} \mathrm{H}_{5}-\mathrm{C} \equiv \mathrm{C}-\mathrm{C} \equiv \mathrm{C}-\mathrm{C}_{6} \mathrm{H}_{5}\right)$ crystallizes from acetic acid in monoclinic prisms. From rotation-, Weissenberg-and Laue-diagrams the symmetry and dimensions of the unit cell have been determined.

$a=6 \cdot 61 \mathrm{~A} . ; b=6 \cdot 04 \mathrm{~A} .: c=14.92 \mathrm{~A} . ; \beta=105^{\circ}$. 